\title{
Endoscopic Internal Thoracic Artery Harvesting with Changes in Scope Position
}

\author{
Yoshihiro Goto, MD, Sho Takagi, MD, PhD, Junji Yanagisawa, MD, Akio Nakasu, MD, Yasuhide Okawa, MD \\ Department of Cardiovascular Surgery, Toyohashi Heart Center, Toyohashi, Japan
}

\section{ABSTRACT}

Although minimally invasive direct coronary artery bypass (MIDCAB) is a less invasive procedure, internal thoracic artery (ITA) harvesting is difficult. A 65 -year-old woman was advised to undergo MIDCAB for recurrent in-stent restenosis. We harvested the ITA using three-dimensional endoscopy without robotics and determined the scope position using enhanced computed tomography. We changed the camera installation between the wound and the camera port, according to the harvesting site with a harmonic scalpel using the skeletonized technique. We harvested the ITA from the subclavian vein level superiorly to the xyphoid process level inferiorly.

\section{INTRODUCTION}

Minimally invasive direct coronary artery bypass (MIDCAB) is a method of coronary artery bypass graft (CABG) surgery that is performed without a median sternotomy. Although MIDCAB is a less invasive procedure, harvesting the internal thoracic artery (ITA) encompasses technical difficulties. The thoracoscopic approach to the ITA takedown has been widely reported since 1994 [Benetti 1995]. In recent years, the usefulness of harvesting the ITA using an endoscope instead of direct vision has been reported, and robotic harvesting is mainly performed [Yang 2015; Gong 2016]. We harvested the ITA through three-dimensional (3D) endoscopy without the robotic system. The procedure was expected to be complicated, due to interference with other instruments and the difficulty of effective visual field development at the specific site. In endoscopic harvesting, the appropriate camera port position is not always evident prior to initiation of the surgery. In this case, we installed the camera into a position between the main wound and the camera port, according to the ITA harvesting site with the harmonic scalpel using the skeletonized technique.

\section{CASE REPORT}

The patient was a 65 -year-old woman with a history of percutaneous coronary intervention for the left anterior

Received August 4, 2021; accepted August 30, 2021.

Correspondence: Yoshibiro Goto, Department of Cardiovascular Surgery, Toyohashi Heart Center, 21 Gobutori, Toyohashi, 4418530, Japan; +81-53237-3377; fax: +81-532-37-3366 (e-mail: yoshibiro.goto57@gmail.com). descending artery (LAD) due to unstable angina two years ago. She was advised to undergo MIDCAB for recurrent instent restenosis. A preoperative enhanced computed tomography (CT) scan demonstrated the view from different aspects (Figure 1). We determined the scope position using enhanced CT. Considering one lung ventilation as ideal, a double-lumen endotracheal tube for single-lung ventilation was used after the induction of anesthesia. The patient was positioned supine with the left side raised using a roll placed longitudinally under the patient. A major 7-cm incision was made in the fifth intercostal space. A $10-\mathrm{mm}$ trocar for a $3 \mathrm{D}$-endoscope was inserted through the fifth intercostal space at the mid-axillary line. The 5 -mm instrumental port was positioned in the fourth intercostal space (Figure 2). Initially, a camera was inserted into the camera port under the collapsed left lung. Once the retractor was positioned, the pleural fat carefully was dissected away to expose the endothoracic fascia covering the left internal thoracic artery (LITA). With total endoscopic vision using 3D endoscopy, the fascia overlying the LITA was scored, and the LITA was taken down with the harmonic scalpel using the skeletonized technique. The LITA was localized and the endothoracic fascia and transverse thoracic muscle were removed from the ITA pedicle for adequate visualization of the vessel. At the distal side of the LITA, the position of the heart obscured the field of vision. We adjusted the camera to a position between the main wound and camera port. There were no obstacles to visualization from the main wound site. The entire length of the mammary artery, starting superiorly at the level of the subclavian vein and ending inferiorly at the level of the
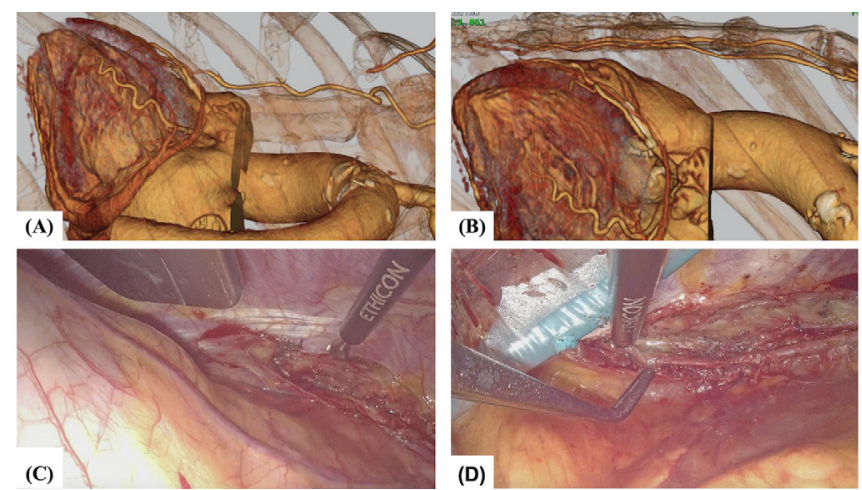

(C)

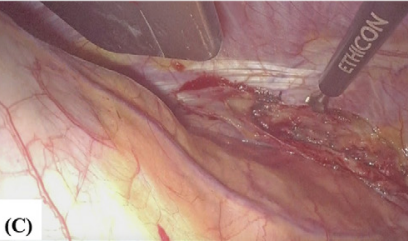

(D)

Figure 1. The distal side of the left internal thoracic artery. (A) View from the camera port during preoperative CT imaging. (B) View from the main incision during preoperative $\mathrm{CT}$ imaging. (C) Operative view from the camera port. (D) Operative view from the site of the main incision. 
(A)

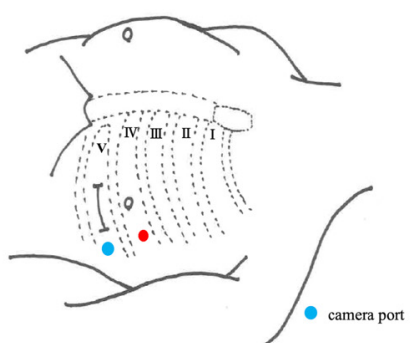

(B)

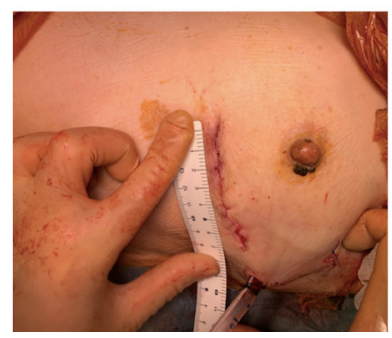

Figure 2. Diagram of the surgical incision and port access position (A). Camera port incision is utilized as a postoperative chest tube site (B).

xyphoid process, was harvested using this technique. Heparin was administered and anastomosis of the coronary artery was performed using a continuous 8-0 polypropylene suture through the main incision. The ITA harvesting time was 56 min, and the operative time was $168 \mathrm{~min}$. The patient had an uneventful postoperative course and was discharged on the fifth postoperative day (Figure 3).

\section{DISCUSSION}

MIDCAB is a reasonable treatment option for proximal LAD stenosis. Minimal access coronary surgery remains a niche for various reasons [Benetti 1995; Yang 2015; Gong 2016]. Endoscopic mammary takedown using the daVinci robot (Intuitive Surgical, Sunnyvale, CA) was first described by Loulmet in 1999 [Loulmet 1999], and Srivastava et al. [Srivastava 2010] reported good results in a large number of cases. We harvested the ITA through 3D endoscopy without the robotic system; 3D endoscopy offers the advantage of a lower running cost than the robotic system [Itagaki 2013]. The 3D endoscope has an enhanced ability of image analysis and enables more reliable visualization of the branches of the ITA than direct vision. Endoscopic harvesting improves short-term outcomes compared with MIDCAB grafting. However, the procedure is expected to be complicated due to interference with other instruments and the difficulty of achieving an effective visual field at the specific site. Our technique helps to avoid interactions between the scope and instruments, during $3 \mathrm{D}$ endoscopic surgery. The camera port positioning has been reported at several sites, including the fourth intercostal space at the mid-clavicular line or the fifth intercostal space at the posterior axillary line [Loulmet 1999; Itagaki 2013; Tomita 2006]. The appropriate position for the camera has been discussed, but not yet determined. In this case, preoperative enhanced CT was performed to detect the coronary artery lesion. We determined the camera port position using preoperative CT. This time, we installed the camera into a position between the main wound and the camera port, according to the ITA harvesting site using the skeletonized technique with the harmonic scalpel. This method enabled us to secure an adequate viewing field without creation of an additional port site.

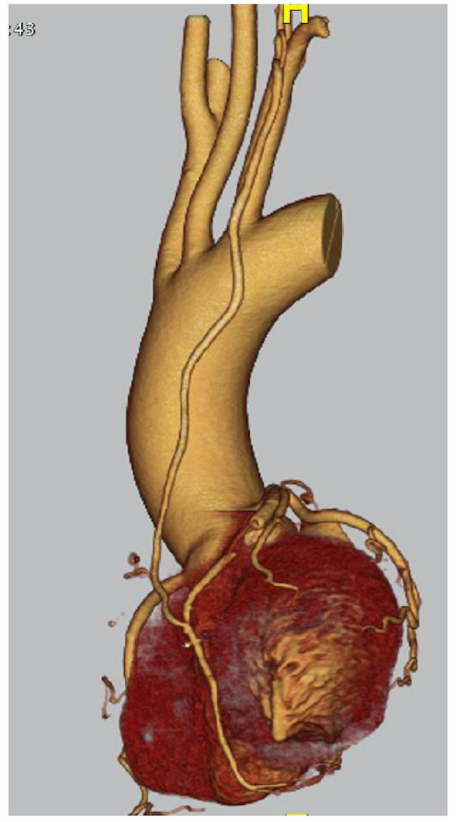

Figure 3. Postoperative enhanced computed tomography scan reveals patent graft.

\section{CONCLUSION}

Harvesting the ITA by 3D endoscopy facilitates delicate manipulation and clear visualization of its proximal and distal sides. Repositioning of the endoscope and instruments according to the harvesting site can make the ITA harvesting procedure easier and safer.

\section{REFERENCES}

Benetti FJ, Ballester C. 1995. Use of thoracoscopy and a minimal thoracotomy, in mammary-coronary bypass to left anterior descending artery, without extracorporeal circulation. Experience in 2 cases. J Cardiovasc Surg. 36:159-61.

Gong W, Cai J, Wang Z, et al. 2016. Robot-assisted coronary artery bypass grafting improves short-term outcomes compared with minimally invasive direct coronary artery bypass grafting. J Thorac Dis. 8:459-68.

Itagaki S, Reddy RC. 2013. Options for left internal mammary harvest in minimal access coronary surgery. J Thorac Dis 5 Suppl. 6:S638-40.

Loulmet D, Carpentier A, d'Attellis N, et al. 1999. Endoscopic coronary artery bypass grafting with the aid of robotic assisted instruments. J Thorac Cardiovasc Surg. 118:4-10.

Srivastava S, Gadasalli S, Agusala M, et al. 2010. Beating heart totally endoscopic coronary artery bypass. Ann Thorac Surg. 89:1873-80.

Tomita S, Watanabe G, Tabata S, et al. 2006. Total endoscopic beatingheart coronary artery bypass grafting using a new 3D imaging system. Innovations. 1:243-6.

Yang M, Wu Y, Wang G, et al. 2015. Robotic total arterial off-pump coronary artery bypass grafting: seven-year single-center experience and long-term follow-up of graft patency. Ann Thorac Surg. 100:1367-73. 